\title{
Cross-Domain Knowledge Transfer for Incremental Deep Learning in Facial Expression Recognition
}

\author{
Nehemia Sugianto and Dian Tjondronegoro
}

\begin{abstract}
For robotics and AI applications, automatic facial expression recognition can be used to measure user's satisfaction on products and services that are provided through the human-computer interactions. Large-scale datasets are essentially required to construct a robust deep learning model, which leads to increased training computation cost and duration. This requirement is of particular issue when the training is supposed to be performed on an ongoing basis in devices with limited computation capacity, such as humanoid robots. Knowledge transfer has become a commonly used technique to adapt existing models and speed-up training process by supporting refinements on the existing parameters and weights for the target task. However, most state-of-the-art facial expression recognition models are still based on a single stage training (train at once), which would not be enough for achieving a satisfactory performance in real world scenarios. This paper proposes a knowledge transfer method to support learning using cross-domain datasets, from generic to specific domain. The experimental results demonstrate that shorter and incremental training using smaller-gap-domain from crossdomain datasets can achieve a comparable performance to training using a single large dataset from the target domain.
\end{abstract}

\section{INTRODUCTION}

Facial expression plays important role in human communication as it can provide a more spontaneous reaction to a particular event and the surrounding environment at a time. Around $55 \%$ of the intended emotion in a spoken message is expressed through facial expression [1]. In terms of customer satisfaction, around $70-95 \%$ unhappy customers tend to show unhappy emotion instead of giving verbal complaint [2]. Hence, by capturing customers' reaction through their facial expression, a robot or AI application can collect useful information if their customers are happy or not with the product or service after they experience it.

One of key challenges in training a deep model for facial expression is the availability of a large-scale facial expression dataset that represent various expression in real world. To date, AffectNet [3] is still the largest 'in-the-wild' facial expression dataset consisting of around 1 million spontaneous facial expression images. The data was collected from the Internet to sufficiently represent real-world scenarios, as well as varieties of face pose, occlusion and ethnicity. The drawback of training on a large-scale dataset is very high training cost in terms of training time and the tendency to require high-performance computing machine.

Nehemia Sugianto is with Department of Business Strategy and Innovation, Griffith University, Queensland 4215, Australia (e-mail: nehemia.sugianto@griffithuni.edu.au).

Dian Tjondronegoro is with Department of Business Strategy and Innovation, Griffith University, Queensland 4215, Australia (e-mail d.tjondronegoro@griffith.edu.au).
This makes deep learning currently unsuitable for small machine with limited computing capabilities, including humanoid robots.

Knowledge transfer is a well-known approach to train a model without training from scratch and offers shorter and cheaper training cost compared to training-at-once and from scratch. The idea is to reuse an existing model, which was trained on particular task and adapt the weights on new training dataset for targeted task. By leveraging and exploiting existing knowledge from different but related domain, we train a model with much less training dataset. Thus, training cost can be greatly reduced as the model only needs to learn from less samples. When properly applied, knowledge transfer can outperform training from scratch (of deep learning model) that is specifically designed for the target task [4]. Furthermore, transfer learning is not only limited to similar source-target domain problem only (homogeneous transfer learning), but also different sourcetarget domain problem (heterogeneous transfer learning). Thus, this approach seems promising to train a facial expression model in an efficient way.

Recent development of facial expression datasets gives us more opportunities to develop better facial expression models and also makes us more selective to choose which the most suitable for our task. The datasets vary in terms of number of size (from small-scale to large-scale), emotion type (from posed to spontaneous), and domains (from in-the-lab domain to in-the-wild domain). The availability of well-known largescale datasets such as ImageNet, VGGFace 2 also add more possibility to explore the benefit of using existing knowledge for facial expression task. Thus, our challenge in transferring knowledge is to determine whether transfer learning using cross-domain dataset with imbalanced distribution between previous and the target domain can still yield a better performance than a training-at-once approach.

This paper proposes a fine-tuning method to transfer knowledge from cross-domain datasets for facial expression recognition task using shorter and incremental training. By combining data across different domains, an existing model can be adapted to a target domain even with an imbalanced proportion of the training datasets.

\section{RELATED WORKS}

Transfer learning approaches can be divided into two categories based on the domain similarities: homogeneous and heterogeneous [5]. In homogeneous transfer learning, the source and target domain have similar feature spaces. In heterogeneous transfer learning, the source and target domains have different feature spaces (i.e. generally nonoverlapping) and the dataset may not share any similarity in 
terms of the labels and feature values. These approaches have been adopted improve the performance and shorten the training time, particularly when the target domain's dataset is limited. In [6], a mixed homogenous and heterogeneous transfer training is used for spontaneous facial expression recognition tasks. Knowledge is initially transferred from a generic object detection dataset (ImageNet), followed by a combination of various facial expression datasets, as the target dataset is very limited. Similar work is also conducted in [7], but this work aims to perform neutral, positive and negative facial expression in video surveillance domain due to the difficulty to collect datasets in the surveillance domain. Based on a similar reason, transfer learning is adopted to transfer knowledge from macro facial expression dataset to recognize micro facial expression, due to the limited dataset in micro facial expression domain. In [8], knowledge transfer is used to build a model that can recognize facial expression using a very small network and small input image.

Despite previous works have demonstrated the feasibility of cross-domain knowledge transfer, there is still a need to further investigate how to achieve an optimal initial model. An optimal deep learning model should be trained with the most suitable datasets to extract a generic knowledge that can be quickly adapted to learn the target task via transfer learning. As the result, we will be able to adapt the model using less samples, which means less computation requirements and faster training time.

\section{METHOD}

Generally, a deeper neural network is required to better recognize the subtle features in facial expressions. We adopt ResNet50 [9] as our base model. Compared to other variants, ResNet50, ResNet101 and ResNet152 have been shown to be more accurate to recognize subtle features [9]. ResNet50 has comparable performance but fewer number of parameters compared to other deeper models. It has $0.85 \mathrm{M}$ parameters and $0.96 \%$ in difference in terms of accuracy compared to deeper models. This makes ResNet50 is suitable when considering memory and training time constraints.

The network consists of five convolutional layers and shortcut connection is applied in the last four layers. Shortcut connection aims to strengthen features by passing important information to the next layers from previous layers. This makes degradation problem can be reduced. To prevent overfitting, down sampling is applied in the last three layers. Lastly, a global average pool is placed at the end and then followed by fully-connected layer for classification purpose.

Strategy to transfer knowledge from generic to specific domain. Fig. 1 illustrates the overall process for the proposed cross-domain knowledge transfer. First, we use datasets from three different domains: generic object detection domain, face recognition domain and facial expression recognition domain to build the initial model. Next, we use various facial expression recognition datasets to learn sufficient variations to tackle the subtle features for recognizing spontaneous and in-the-wild facial expressions, as represented by the AffectNet dataset for the target domain.

There are six models presented to investigate how knowledge from cross-domain can be transferred effectively

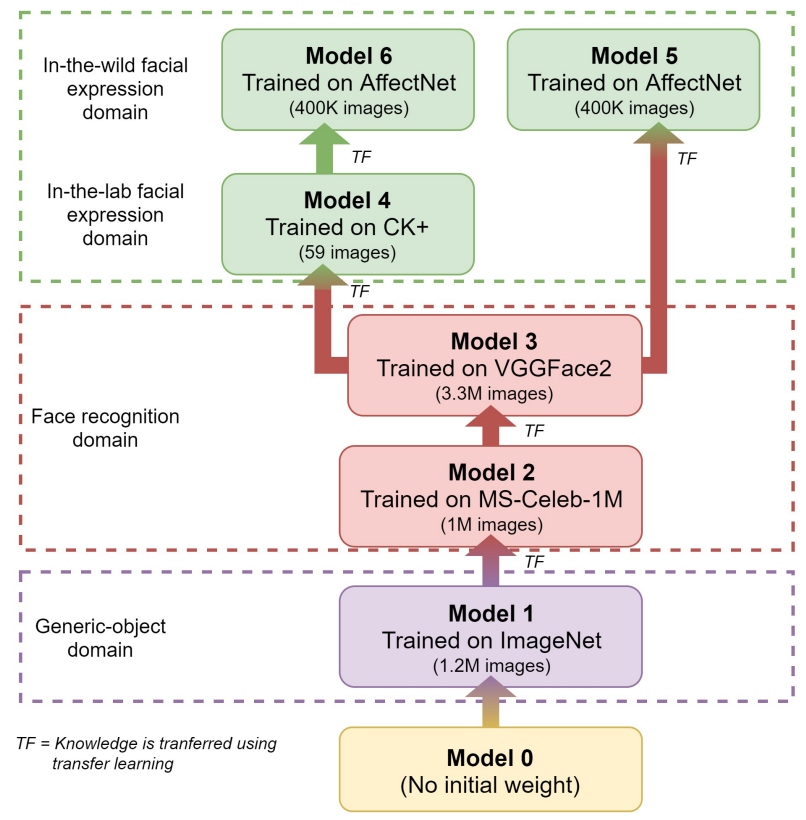

Figure 1. Proposed incremental training scheme

towards the targeted task. Model 0 is built without any prior knowledge. For initial knowledge gained from cross-domain, Model $1-3$ are constructed using both different and similar domains (generic-object domain and face recognition domain), representing a heterogeneous knowledge transfer. Then, Model 4-5 are constructed from Model 1-3 by training them using facial expression datasets, which is the target domain.

Knowledge learned from the previous datasets can be transferred by unfreezing some layers of the network to be trained on the new datasets. This will make the model's parameters and parameters become increasingly adapted for the target domain. A key consideration is how far we can adapt the layer. In many cases, adapting all layers can work well when we have enough dataset [10]. However, when we have a very limited dataset, unfreezing too many layers might result losing important information that the model has learned.

Dataset imbalance. AffectNet [3] has an imbalanced dataset issue. Some emotions (e.g. sad, surprise, fear, disgust, anger, contempt) have less samples than the average number of samples ( $35 \mathrm{~K}$ images). Happy and neutral are emotions with the highest samples $(\sim 133 \mathrm{~K}$ and $\sim 74 \mathrm{~K}$ images respectively). This condition can affect the model's overall performance during training. Emotions with less samples will have lower possibility to converge, as the model is harder to learn the emotion due to not enough sample. On the other hands, emotions with more samples most likely have bigger possibility to perform better.

To address this issue, down-sampling, up-sampling, and weighted-loss approaches can be adopted to make the dataset more balanced. Down-sampling is selecting certain number of samples for each class so all classes will have exact number of samples. Up-sampling is replicating samples in some emotion classes so all classes will have exact number of samples. Weighted-loss is applying weight on the loss 
function for each emotion class relatively to the size proportion in the dataset. In our experiments, down-sampling is applied. The result is still a semi-balanced dataset as shown in Table 1, as disgust, contempt and fear are the specific emotion classes that have the most $(15,000)$ samples, while the other classes still have less samples.

TABLE I. DATASET Distribution on AfFectNet Using DOWN-SAMPLING IN ORDER TO BALANCE THE DATASET

\begin{tabular}{|c|c|c|c|c|c|c|}
\hline Emotion & \multicolumn{2}{|c|}{ \#Train } & \multicolumn{2}{|c|}{ \#Val } & \multicolumn{2}{|c|}{ \#Test } \\
\hline Neutral & 15,000 & $18 \%$ & 600 & $13 \%$ & 500 & $13 \%$ \\
\hline Happy & 15,000 & $18 \%$ & 600 & $13 \%$ & 500 & $13 \%$ \\
\hline $\mathrm{Sad}$ & 15,000 & $18 \%$ & 600 & $13 \%$ & 500 & $13 \%$ \\
\hline Surprise & 15,000 & $18 \%$ & 600 & $13 \%$ & 500 & $13 \%$ \\
\hline Fear & 11,556 & $13 \%$ & 600 & $13 \%$ & 500 & $13 \%$ \\
\hline Disgust & 6,404 & $7 \%$ & 600 & $13 \%$ & 500 & $13 \%$ \\
\hline Anger & 15,000 & $18 \%$ & 600 & $13 \%$ & 500 & $13 \%$ \\
\hline Contempt & 6,300 & $7 \%$ & 600 & $13 \%$ & 500 & $13 \%$ \\
\hline & 99,262 & & 4,800 & & 4,000 & \\
\hline
\end{tabular}

Noise reduction. Facial expression recognition task is more sensitive to noise compared to object recognition task as facial expression is located mostly on eyes and mouth and forms a small fraction of a frontal face image [11]. Therefore, any non-facial region (e.g. neck, hair, and background) can be seen as noise, which is unimportant or unrelated data for the model to learn and can degrade the model performance and slowing down the model's learning speed.

One approach to reduce noise is by cropping the facial region as closely as possible, removing any unnecessary parts outside of the crucial facial action points. The key challenges is how to avoid accidentally removing the important features if the cropping is inaccurate or too far. In our experiments, the training images are provided in three different formats: uncropped, outer-cropped, and inner-cropped. Outer-cropped is obtained by cropping the facial region based on all facial landmark points whereas inner-cropped is obtained by cropping the facial region based on facial landmarks points excluding the face contour (facial landmark point 1 - 17). Uncropped means using original images provided by the authors (no facial region cropping is performed). Fig. 2 shows the different results of the facial region cropping.

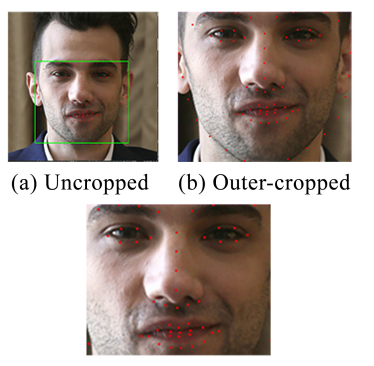

(c) Inner-cropped

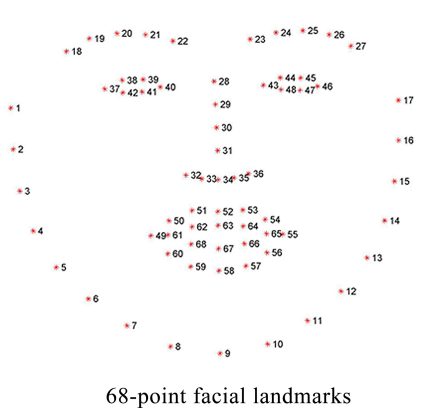

68-point facial landmarks
Figure 2. Different types of facial region cropping based on facial landmarks

\section{EXPERIMENTAL SETUP}

\section{A. Datasets}

The experiments use four datasets from different domains: ImageNet, Microsoft Celeb (MS-Celeb-1M), VGGFace2, $\mathrm{CK}+$, and AffectNet. Four datasets except $\mathrm{CK}+$ are collected from in-the-wild environment (e.g. from Internet) and classified as large-scale datasets. CK+ was collected in a laboratory environment.

ImageNet [12] is a large-scale dataset of generic object images, which is organized according to the WordNet hierarchy. It contains $>1.2 \mathrm{M}$ labelled images depicting 1,000 different object classes including multiple bounding boxes. This dataset has been a proven well-known dataset used in knowledge transfer works.

Microsoft Celeb (MS-CELEB-1M) [13] is a large-scale face dataset used for face recognition purpose. It consists of $10 \mathrm{M}$ face images from nearly $100 \mathrm{~K}$ different celebrities, which was collected from the Internet by Microsoft.

VGGFace2 [14] is another large-scale face dataset collected from Google Image search for face recognition purpose. It consists Net, of $3.3 \mathrm{M}+$ face images from $9 \mathrm{~K}+$ different people. It has large variations in terms of ethnicity, age, emotion, pose illumination, and occlusion conditions.

Extended Cohn-Kanade (CK+) $[15,16]$ is one of wellknown facial expression dataset, which consists of posed and spontaneous emotions. It is an extended version of original CK dataset and composed of 593 sequences across 123 subjects. Each sequence begins with neutral expression and then ends with peak expression. The peak emotion is then classified into seven basic emotions including neutral emotion.

AffectNet [3] is the largest facial expression dataset which contains $\sim 1 \mathrm{M}$ spontaneous facial expression images. It was obtained from the Internet by querying three major search engines using 1,250 emotions related keywords in six different languages. About $\sim 420 \mathrm{~K}$ images are manually annotated by human annotators and classified by categorical model (seven discrete facial expression) and dimensional model (intensity of valence and arousal). The rest of the images $(\sim 550 \mathrm{~K})$ are automatically annotated by a residual network which was trained on manually annotated samples.

For facial expression datasets, facial region is obtained by the facial landmark points. For $\mathrm{CK}+$, facial region is calculated using FacemarkAAM [17]. For AffectNet, facial region is calculated by facial landmarks provided by the authors.

\section{B. Training and Testing}

Each model is implemented using Tensorflow and trained on a NVIDIA GTX 2080 GPU and takes more than 2 days per experiment as number of trained layers affects the number of hours to train the models. The more layers are trained, the longer time to train the model as it performs more complex computation and requires more memory resource. 
Our models are trained using various hyper parameters and $S G D$ (Stochastic Gradient Descent) as the optimizer which was used originally in ResNet. In general, the decay is $5.10^{-4}$ and momentum is 0.9 using Nesterov momentum. We experimentally determine that $1.10^{-2}$ is the best learning rate in most training cases. The learning rate can be reduced to $1.10^{-3}$ when the validation accuracy is on a plateauing stage with factor of 0.5 and patience is 10 . Each dataset is divided into $90 \%$ for training set ( $20 \%$ is used as validation set), and $10 \%$ for test set. For AffectNet, image distribution is described in Table 1. Sample for each class are mutually exclusive, as no overlapping images are allowed in between different classes.

\section{EXPERIMENTAL RESULTS}

Performance comparison on knowledge transfer from cross-domain datasets. As seen in Table 2, training a model from scratch without prior knowledge has the worst training accuracy in all datasets (see Model 0): 43.87\%, 61.03\%, $59.28 \%(\mathrm{CK}+$, AffectNet, $\mathrm{CK}+\rightarrow$ AffectNet). It shows that training without prior knowledge will make the model harder to converge to the targeted task. Training using CK+ has the lowest performance compared to other datasets due to the small number of samples (less than 100 samples).

Training a model with initial knowledge from genericobject domain (ImageNet) gives much improvement in each dataset up to $49 \%$ (see Model 1). The largest improvement can be seen on Model 1 trained on $\mathrm{CK}+$ from $43.87 \%$ increased to $93.15 \%$. Although $\mathrm{CK}+$ only has very small dataset, but the prior knowledge helps the model to learn the target task much better. Model 1 trained on AffectNet also benefits the prior knowledge to improve the performance from $61.03 \%$ to $87.34 \%$. Although the improvement is not that much compared to $\mathrm{CK}+$, the prior knowledge is still very useful for the model to learn a broad range of variations in the dataset. It can give improvement by $12 \%$ compared to the model without prior knowledge (Model 0 trained on AffectNet). It shows that transferring knowledge from generic-object domain can give positive effect to the model to learn targeted task as it shares low-to-mid feature level (e.g. lines, edges) between generic and facial expression domain.

TABLE II. TRAINING ACCURACY COMPARISON FROM VARIOUS TRAINING ON CROSS-DOMAIN DATASETS

\begin{tabular}{|c|c|c|c|}
\hline & Base model & $\begin{array}{c}\text { Training } \\
\text { dataset }\end{array}$ & $\begin{array}{l}\text { Training } \\
\text { accuracy }\end{array}$ \\
\hline \multirow{4}{*}{$\frac{ \pm}{\frac{ \pm}{0}}$} & Model 0 (No initial weight) & \multirow{4}{*}{$\mathbf{C K}+$} & $43.87 \%$ \\
\hline & Model 1 (IN) & & $89.10 \%$ \\
\hline & Model 2 (IN $\rightarrow$ MC1M) & & $91.48 \%$ \\
\hline & Model $3($ IN $\rightarrow$ MC1M $\rightarrow$ VF2) & & $93.15 \%$ \\
\hline \multirow{4}{*}{$\begin{array}{l}\frac{n}{0} \\
\frac{0}{0} \\
\sum\end{array}$} & Model 0 (No initial weight) & \multirow{4}{*}{ AffectNet } & $61.03 \%$ \\
\hline & Model 1 (IN) & & $73.68 \%$ \\
\hline & Model $2(\mathrm{IN} \rightarrow \mathrm{MC} 1 \mathrm{M})$ & & $82.90 \%$ \\
\hline & Model $3($ IN $\rightarrow$ MC1M $\rightarrow$ VF2) & & $87.34 \%$ \\
\hline \multirow{4}{*}{$\frac{0}{\frac{0}{0}}$} & Model 0 (No initial weight) & \multirow{4}{*}{$\begin{array}{l}\text { CK+, then } \\
\text { followed by } \\
\text { AffectNet }\end{array}$} & $59.28 \%$ \\
\hline & Model 1 (IN) & & $70.39 \%$ \\
\hline & Model 2 (IN $\rightarrow$ MC1M) & & $78.17 \%$ \\
\hline & Model $3($ IN $\rightarrow$ MC1M $\rightarrow$ VF2) & & $83.56 \%$ \\
\hline
\end{tabular}

Grey denotes the best model. IN = ImageNet, MC1M = MS-CELEB-1M, VF2 = VGGFace
Table 3 shows how the performance comparison of model 3 on targeted task (facial expression task on AffectNet dataset). The best performance is achieved when model 3 is trained on AffectNet which is 0.60 for F1 score. It shows that the model is able to learn targeted task and achieves good performance although the model is not trained using all samples. However, when model 3 is trained on $\mathrm{CK}+$ and then followed by AffectNet, the performance only achieves 0.57 for F1 score which is lower when trained only on AffectNet. We believe the number of samples of $\mathrm{CK}+$ causes significant drop when the model learns new domain. The worst performance is achieved when model 3 is trained on $\mathrm{CK}+$ which $\mathrm{F} 1$ score is 0.23 . We believe that $\mathrm{CK}+$ cannot cover all characteristics of AffectNet, which has a very broad range of variations.
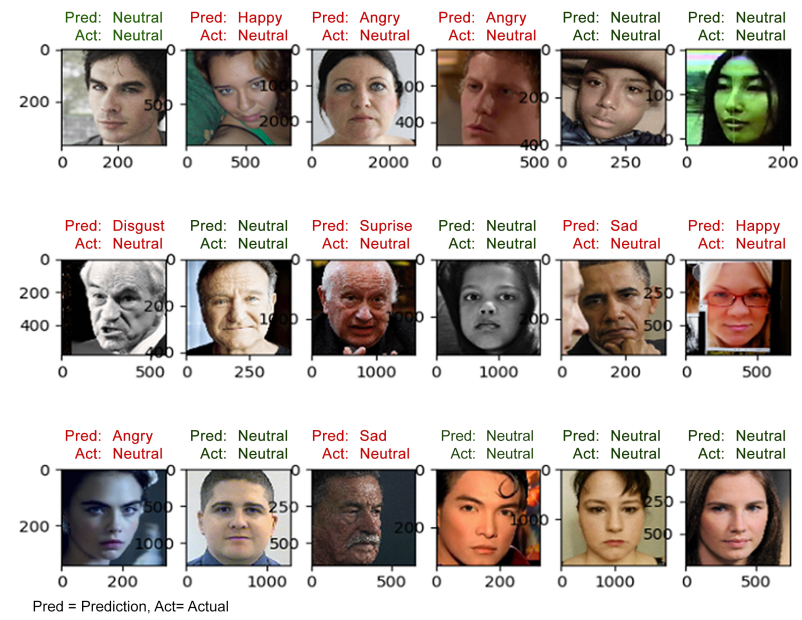

Figure 3. Some samples of facial expression recognition using Model 5 when tested on AffectNet test set

TABLE III. PERFORMANCE COMPARISON WHEN TESTED ON AFFECTNET

\begin{tabular}{|c|l|c|c|c|}
\hline Model & \multicolumn{1}{|c|}{ Datasets } & Prec & Recall & F1 \\
\hline Model 4 & Model 3 $\rightarrow$ CK + & 0.228 & 0.251 & 0.23 \\
\hline Model 5 & Model 3 $\rightarrow$ AffectNet & $\mathbf{0 . 6 1 7}$ & $\mathbf{0 . 6 3 1}$ & $\mathbf{0 . 6 0}$ \\
\hline Model 6 & Model 3 $\rightarrow$ CK $+\rightarrow$ AffectNet & 0.577 & 0.594 & 0.57 \\
\hline
\end{tabular}

Grey denotes the best model

Effect of noise reduction on facial region. Table 4 shows the performance comparison when model 3 trained on AffectNet using different facial region cropping. Training on inner-cropped samples can give the best training accuracy compared to uncropped and outer-cropped facial region with difference up to $8 \%$. It shows that inner-cropped facial region can eliminate noise in facial region which makes the model learn better as we only provide relevant features to learn targeted task.

TABLE IV. PERFormanCE OF MOdEL 3 WHEN Trained ON AFFECTNET With DifFERENT FACIAL REGION CROPPING

\begin{tabular}{|c|c|c|}
\hline Model & Facial region & Training accuracy \\
\hline \multirow{2}{*}{$\begin{array}{c}\text { Model 5 } \\
\text { (Model 3 } \rightarrow \text { AffectNet) }\end{array}$} & Uncropped & $79.40 \%$ \\
\cline { 2 - 3 } & Outer-cropped & $83.56 \%$ \\
\cline { 2 - 3 } & Inner-cropped & $\mathbf{8 7 . 3 4 \%}$ \\
\hline
\end{tabular}




\section{DISCUSSION}

Training on cross-domain datasets with smaller domain gap enables the model to learn the targeted task with less samples. By transferring knowledge from multi-datasets with smaller domain gap in each training, accumulated knowledge is built and prepares the model to be readier when learning targeted task. Model 5 (ImageNet $\rightarrow$ MSCELEB-1M $\rightarrow$ VGGFace2 $\rightarrow$ AffectNet) gives the best performance when knowledge is transferred in closer gap domain manner; from generic-object domain to face recognition domain and then facial expression domain, compared to no prior knowledge transferred (Model 0) and generic-object domain transferred (Model 1). As a result, the model can easily converge to the targeted task with less samples and short training time. Fig.5 shows some samples of facial expression recognition using Model 5 on AffectNet test set.

\section{CONCLUSION AND FUTURE WORKS}

This paper extends the concept of knowledge transfer from cross-domain datasets to enable the model learn target task with less samples. By exploiting knowledge from existing large-scale dataset from different domain, we have combined homogeneous and heterogeneous knowledge transfer approaches for facial expression task. The experimental results confirm that the accumulated knowledge transferred from cross-domain with small domain gap enables the model to learn targeted task with less samples with comparable result (F1 score of 0.60) compared to training using all samples conducted originally in (F1 score of 0.62) [3]. The use of face cropping is also important to improve performance, due to noise reduction in features extraction of the deep learning models.

Future work will explore various knowledge transfer methods, beyond fine-tuning, in order to better understand the effects of each method on optimizing the performance. We also need to investigate further strategies to address data imbalance and noise reduction to continue improving the method. Furthermore, a deployment on a small device, such as a humanoid robot, will confirm the practical benefits of using the proposed incremental learning. Facial expression recognition needs to adapt to context and environment. The more that the model is customized for the specific target domain and user groups, the more accurate it will be. In robotic applications, this means that a robot's ability to better understand the users' emotions would be enhanced after it interacts and learns from more instances of the target domain

\section{REFERENCES}

[1] McKinsey\&Company, "Customer Experience: Creating Value through Transfroming Customer Journeys,"

[2] O. Harari, "Thank heaven for complainers," Management Review, vol. 81, no. 1, p. 59, 1992.

[3] A. Mollahosseini, B. Hasani, and M. H. Mahoor, "Affectnet: A database for facial expression, valence, and arousal computing in the wild," arXiv preprint arXiv:1708.03985, 2017.

[4] B. Tan, Y. Zhang, S. J. Pan, and Q. Yang, "Distant domain transfer learning," in Thirty-First AAAI Conference on Artificial Intelligence, 2017.
[5] O. Day and T. M. Khoshgoftaar, "A survey on heterogeneous transfer learning," Journal of Big Data, vol. 4, no. 1, p. 29, 2017.

[6] H.-W. Ng, V. D. Nguyen, V. Vonikakis, and S. Winkler, "Deep learning for emotion recognition on small datasets using transfer learning," in Proceedings of the 2015 ACM on international conference on multimodal interaction, 2015, pp. 443-449: ACM.

[7] N. Sugianto, D. Tjondronegoro, and B. Tydd, "Deep Residual Learning for Analyzing Customer Satisfaction using Video Surveillance," in 2018 15th IEEE International Conference on Advanced Video and Signal Based Surveillance (AVSS), 2018, pp. 1-6: IEEE.

[8] İ. Çuğu, E. Şener, and E. Akbaş, "MicroExpNet: An Extremely Small and Fast Model For Expression Recognition From Frontal Face Images," arXiv preprint arXiv:1711.07011, 2017.

[9] K. He, X. Zhang, S. Ren, and J. Sun, "Deep residual learning for image recognition," in Proceedings of the IEEE conference on computer vision and pattern recognition, 2016, pp. 770-778.

[10] Y. Deng et al., "Knowledge as A bridge: Improving cross-domain answer selection with external knowledge," in Proceedings of the 27th International Conference on Computational Linguistics, 2018, pp. 3295-3305.

[11] P. Ekman and E. L. Rosenberg, What the face reveals: Basic and applied studies of spontaneous expression using the Facial Action Coding System (FACS). Oxford University Press, USA, 1997.

[12] O. Russakovsky et al., "Imagenet large scale visual recognition challenge," International journal of computer vision, vol. 115, no. 3, pp. 211-252, 2015.

[13] Y. Guo, L. Zhang, Y. Hu, X. He, and J. Gao, "Ms-celeb-1m: A dataset and benchmark for large-scale face recognition," in European Conference on Computer Vision, 2016, pp. 87-102: Springer.

[14] Q. Cao, L. Shen, W. Xie, O. M. Parkhi, and A. Zisserman, "Vggface2: A dataset for recognising faces across pose and age," in 2018 13th IEEE International Conference on Automatic Face \& Gesture Recognition (FG 2018), 2018, pp. 67-74: IEEE.

[15] T. Kanade, J. F. Cohn, and Y. Tian, "Comprehensive database for facial expression analysis," in Automatic Face and Gesture Recognition, 2000. Proceedings. Fourth IEEE International Conference on, 2000, pp. 46-53: IEEE.

[16] P. Lucey, J. F. Cohn, T. Kanade, J. Saragih, Z. Ambadar, and I. Matthews, "The extended cohn-kanade dataset $(\mathrm{ck}+)$ : A complete dataset for action unit and emotion-specified expression," in Computer Vision and Pattern Recognition Workshops (CVPRW), 2010 IEEE Computer Society Conference on, 2010, pp. 94-101: IEEE.

[17] G. Tzimiropoulos and M. Pantic, "Optimization problems for fast aam fitting in-the-wild," in Proceedings of the IEEE international conference on computer vision, 2013, pp. 593-600. 